\title{
Dynamic Electropulsing Induced Phase Transformations and Their Effects on Single Point Diamond Turning of AZ91 Alloy
}

\author{
Duo Zhang ${ }^{1}$, S. To ${ }^{1 *}$, Yaohua Zhu ${ }^{*}$, Hao Wang ${ }^{1}$, Guoyi Tang ${ }^{2}$ \\ ${ }^{1}$ State Key Laboratory in Ultra-Precision Machining Technology, Department of Industrial and Systems Engineering, Hong Kong \\ Polytechnic University, Hong Kong, China; ${ }^{2}$ Gratduate School at Shenzhen, Tsinghua University, Shenzhen, China. \\ Email: ${ }^{*}$ mfsto@inet.polyu.edu.hk, yaohuazhu@hotmail.com
}

Received November $4^{\text {th }}, 2011$; revised December $9^{\text {th }}, 2011$; accepted December $23^{\text {rd }}, 2011$

\begin{abstract}
The effects of dynamic electropulsing on microstructure changes and phase transformations of a rolled Mg-9Al-1Zn alloy were studied by using optical microscopy, X-ray diffraction, back-scattered scanning microscopy and transmission electron microscopy techniques. The decomposition of $\beta$ phase was accelerated under dynamic electropulsing, compared with the conventional thermal processes. Dynamic electropulsing was less effective in affecting the phase transformations, but more effective in reducing residual stress than the static electropulsing. Dynamic electropulsing improved machinability of single point diamond turning, the mechanism of which is discussed from the point of view of dislocation dynamics.
\end{abstract}

Keywords: Electropulsing; Phase Transformations; Dislocation; Twins; Magnesium Alloy

\section{Introduction}

Alloy materials suffer various external stresses during their manufacturing and in subsequent service [1]. It is of significant practical importance to reduce these residual stresses. It was reported that under electropulsing treatment (EPT), the residual stress was reduced significantly [2-10]. There are two practical types of electropulsing: static electropulsing and dynamic electropulsing. The former combines a thermal process with electropulsing. The latter is a complex thermal process, which combines simultaneously both electropulsing and plastic deformation.

In the present work, the dynamic electropulsing induced phase transformations and microstructural changes, and their effects on Single Point Diamond Turning (SPDT) of the AZ91 alloy are studied from the point view of dislocation dynamics.

\section{Experimental Procedures}

A commercial magnesium alloy AZ91 $(9.1 \mathrm{wt} \% \mathrm{Al}$, $0.9 \mathrm{wt} \% \mathrm{Zn}, 0.2 \mathrm{wt} \% \mathrm{Mn}$, balance $\mathrm{Mg}$ ) was used. The ingot was homogenized at $693 \mathrm{~K}$ for $16 \mathrm{hrs}$ and subsequently extruded into strip of $2.90 \mathrm{~mm}$ wide and 1.45 $\mathrm{mm}$ thick. The extruded strip was then rolled under electropulsing. During the rolling, the strip was moving at a

\footnotetext{
"Corresponding author.
}

speed of $2 \mathrm{~m} / \mathrm{min}$ between two electrodes set at a distance of $225 \mathrm{~mm}$. The thicknesses of the strip specimens before and after rolling were $1.45 \mathrm{~mm}$ and $1.20 \mathrm{~mm}$ thick, respectively. A self-made electropulsing generator was continuously applied to discharge multiple positive pulses with various current parameters on the rolling material, and it took about 10s for the strip to pass through the electrodes. During the dynamic electropulsing, the surface temperature was measured by a contact thermocouple for each test, and the current parameters including frequency, root-mean-square current (RMS), amplitude current and duration of multiple pulses were monitored by an oscilloscope connected with a Hall effect sensor. These are listed in Table 1. A schematic illustration of the dynamic electropulsing system is shown in Figure 1.

Longitudinal cross-sections of the specimens with various frequencies of EPT were polished before being examined under an electron microscope. A HitachiS4800 ultra-high resolution field emission scanning electron microscope was used in a back-scattered electron mode (BSEM) for examination of the microstructure of the specimens. The polished specimens were etched with a commercial solution [9] before the metallographic observation using an Olympus GX51 Inverted Microscope. An X-ray diffraction examination was performed with an X-ray diffractometer (Bruker D8 Advance), using nickel- 
Table 1. Operation parameters of the electropulsing.

\begin{tabular}{lccccc}
\hline \multicolumn{2}{c}{ No. } & Frequency $(\mathrm{Hz})$ & $\begin{array}{c}\text { Duration } \\
(\mu \mathrm{s})\end{array}$ & RMS (A) & $\begin{array}{c}\text { Amplitude } \\
\left(\mathrm{A} / \mathrm{mm}^{2}\right)\end{array}$ \\
\hline EPT 1 & Non-EPT & & & & 293 \\
EPT 2 & 126 & 70 & 52.00 & 211.2 & 316 \\
EPT 3 & 204 & 70 & 63.05 & 237.6 & 349 \\
EPT 4 & 265 & 70 & 89.53 & 256.5 & 359 \\
EPT 5 & 309 & 70 & 100.19 & 271.6 & 390 \\
\hline
\end{tabular}

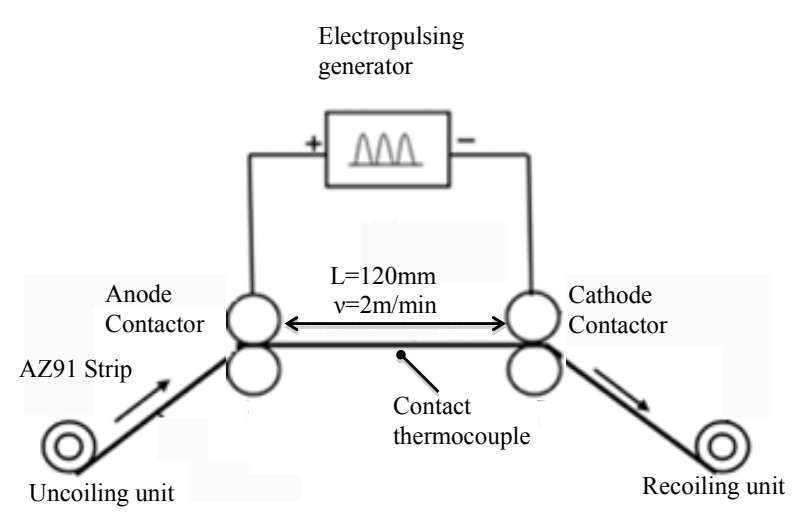

Figure 1. A schematic view of dynamic electropulsing process.

filtered $\mathrm{Cu} K_{\alpha}$ radiation. The range of diffraction was selected from $25^{\circ}$ to $65^{\circ}(2 \theta)$. The scanning speed was 1 degree/min. An examination of TEM was carried out using a JEOL 2010 transmission electron microscope. A Gatan 691 Precision Ion Polishing System (PIPS) was used in the preparation of a thin film of the alloy for TEM examination.

After EPT, SPDT was performed on a two-axis computer numerically controlled (CNC) precision single point diamond turning machine (OptoForm 30 SPDT machine), and a face turning model of SPDT was used. The machining parameters are listed in Table 2. A force transducer Kistler 9252A was mounted with a pre-loaded force under the tool shank. A 14-bit multifunctional data acquisition (DAQ) card PCI-6132 (National Instrument) was configured on a PC workstation to record the data of the cutting force.

\section{Results and Discussion}

\subsection{Electropulsing Induced Phase Transformations and Microstructure Changes}

Figure 2 shows the X-ray diffractograms of the as-rolled (non-EPT) specimen and the dynamic EPT specimens. The non-EPT specimen consisted of mainly two phases,
Table 2. Operating parameters of face turning in SPDT.



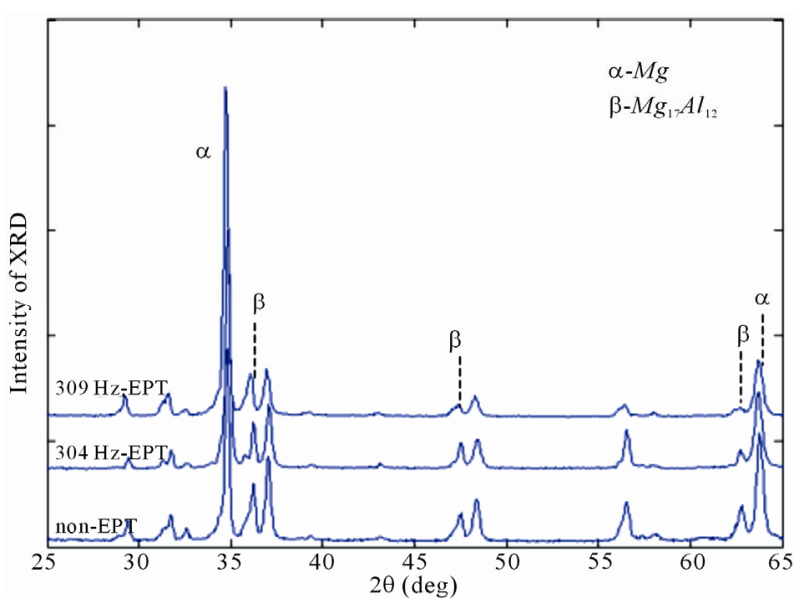

Figure 2. XRD patterns for specimens under various frequencies of electropulsing.

$\alpha$-Mg phase and a $\beta$-phase $\left(\mathrm{Mg}_{17} \mathrm{Al}_{12}\right)$. When the frequency of electropulsing increased to $204 \mathrm{~Hz}$, the XRD intensity of $\beta$ phase reduced. Upon further increasing the frequency to $309 \mathrm{~Hz}$, the XRD intensity of $\beta$ phase further reduced. That implies the decomposition of $\beta$ phase was accelerated when the frequency of electropulsing increased during dynamic electropulsing.

The BSEM images and TEM bright field images of specimens under various frequencies of dynamic EPT are shown in Figure 3. The matrix was $\alpha-\mathrm{Mg}$ phase, while the precipitates were the $\beta$-phase $\left(\mathrm{Mg}_{17} \mathrm{Al}_{12}\right)$. For the as rolled specimen, numerous precipitates of $\beta$-phase can be found in Figure 3(a1); as the frequency of dynamic electropulsing increased to $204 \mathrm{~Hz}$, the amounts of $\beta$-phase precipitates decreased as shown in Figure 3(a2). When the frequency of electropulsing was increased to $309 \mathrm{~Hz}$, even fewer precipitates could be observed, as shown in Figure 3(a3). TEM bright field images of these specimens confirmed the same microstructure changes as shown in Figures 3(b1), (b2) and (b3). As the frequency of electropulsing increased, the amounts of precipitates marked by the white arrows decreased significantly, meaning that the decomposition of $\beta$ phase occurred simultaneously. Both the SEM results and TEM observations were in good agreement with the XRD patterns, which are shown in Figure 2.

The TEM examination of $\beta$ phase is shown in Figure 4. The bright field of the $204 \mathrm{~Hz}-\mathrm{EPT}$ specimen is shown 


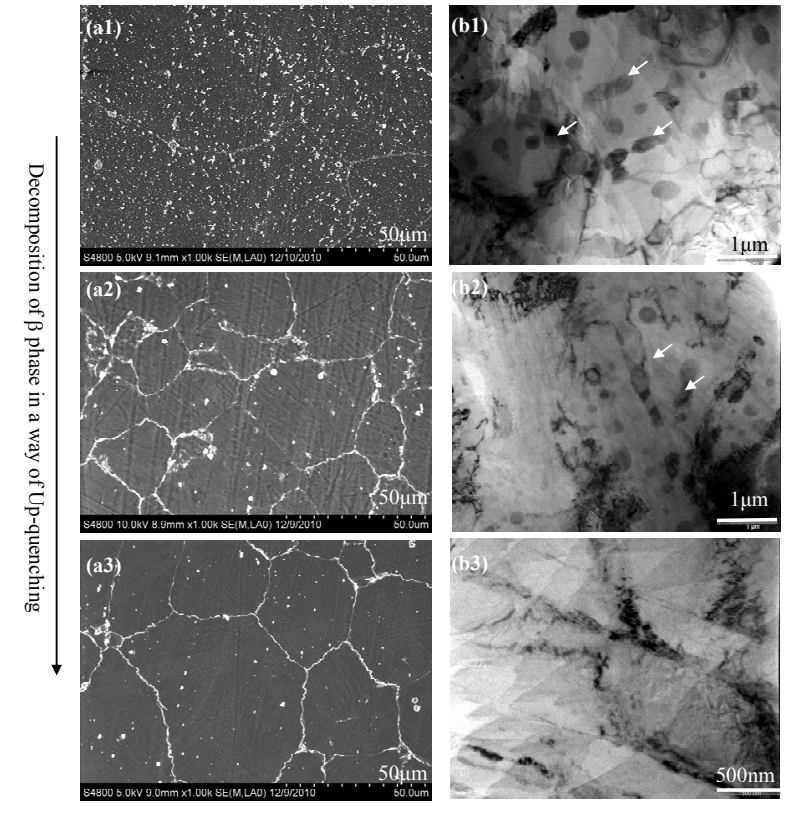

Figure 3. BSEM images of specimens: (a1) Non-EPT; (a2) $204 \mathrm{~Hz}-\mathrm{EPT}$; (a3) $309 \mathrm{~Hz}-\mathrm{EPT}$ and TEM bright field images of specimens: (b1) Non-EPT; (b2) 204 Hz-EPT; (b3) 309 Hz-EPT, showing $\beta$ phase precipitates changes with various frequencies of dynamic electropulsing.



Figure 4. TEM images of specimens after $204 \mathrm{~Hz}$ EPT: (a) Bright field image; (b2) Dark field image of $(10 \overline{10})$ reflection together with (b1) diffraction pattern from $B=[\overline{12} \overline{1} 3]$ of the $\alpha$ phase; (c2) Dark field image of (110) reflection together with (c1) diffraction pattern from $B=[\overline{111}]$ of the $\beta$ phase. in Figure 4(a). Together with the indexed SADPs shown in Figures 4(b1) and (c1), the dark field images reflected from $[\overline{12} \overline{1} 3](10 \overline{10})$ of the hep $\alpha$ phase, and $[\overline{11} 1]$ (110) the bcc $\beta$ phases are shown in Figures 4(b2) and (c2), respectively. The lattice parameters of the phase are $a=3.22 \AA, c=5.23 \AA$ and $c / a=1.624$; the lattice parameters of the $\beta$ phase is $a=10.690 \AA$.

For comparison, the AZ91 strips were statically electropulsing-treated under various parameters. The BSEM images and TEM bright field images of the static EPT specimens are shown in Figure 4. It was reported that with increasing frequency of electropulsing [12], decomposition (in a way of up-quenching) and precipitation (in a way of quenching) of $\beta$ phase were tremendously accelerated sequentially, shown in Figure 5. While in the dynamic EPT specimens, only the decomposition of $\beta$ phase by a way of up-quenching occurred, as shown in Figure 3. The decomposition of $\beta$ phase occurred after static $105 \mathrm{~Hz}$-EPT for $10 \mathrm{~s}$, while that completed after dynamic $309 \mathrm{~Hz}-\mathrm{EPT}$ for $10 \mathrm{~s}$. A higher frequency of electropulsing was needed in order to complete the decomposition of $\beta$ phase in the same duration of electropulsing in the dynamic EPT alloy specimens.

It has been reported that for the decomposition to complete under conventional thermal process of the AZ91 strips, 10 hours of ageing in the range of $663 \mathrm{~K}$ was required [11]. This implies that electropulsing tremendously accelerated the phase transformations of the

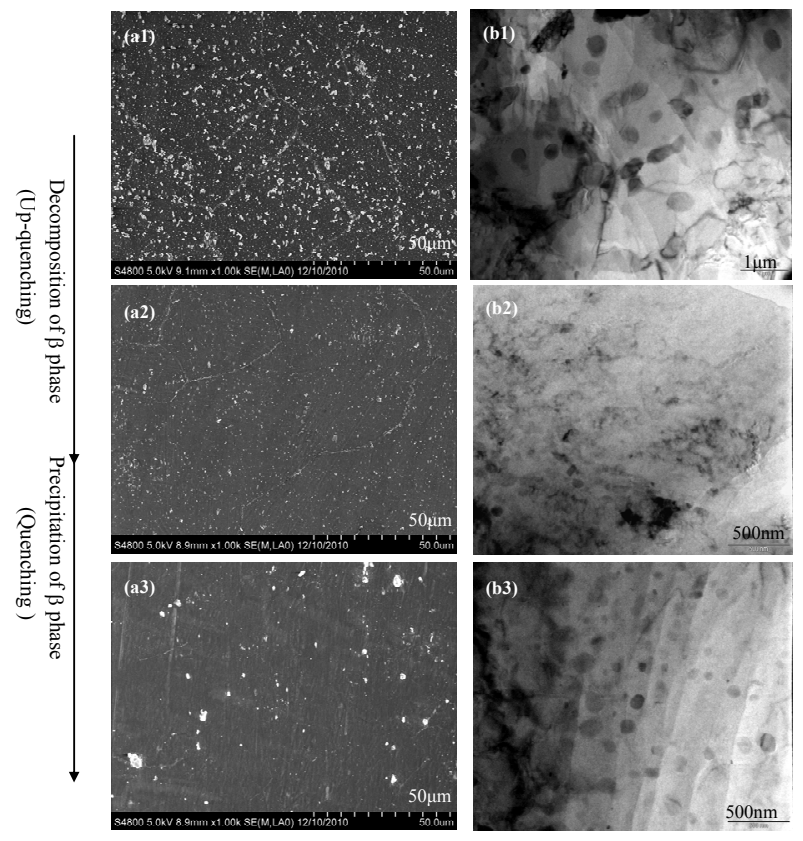

Figure 5. BSEM images of specimens: (a1) Non-EPT; (a2) $151 \mathrm{~Hz}-E P T$; (a3) $294 \mathrm{~Hz}-\mathrm{EPT}$ and TEM bright field images of specimens: (b1) Non-EPT; (b2) 151 Hz-EPT; (b3) 294 Hz-EPT, showing changes of $\beta$ phase precipitate with various frequencies of static electropulsing. 
AZ91 alloy at a relatively lower temperature within only a few seconds.

The optical micrographs of the non-EPT and the dynamic EPT specimens with various frequencies are shown in Figure 6. For the non-EPT specimen, plenty of deformation twins were observed inside the grains, as shown in Figure 6(a). When the frequency of dynamic electrouplsing increased to $126 \mathrm{~Hz}$, the twins inside the grains were rarely observed, as shown in Figure 6(b). Upon further increasing the frequency to $309 \mathrm{~Hz}$, a relatively homogeneous microstructure of equal-axed grains was obtained, as shown in Figures 6(c)-(e). This was because under the dynamic electropulsing the external stress that resulted from the plastic deformation was reduced simultaneously, and the twins quickly vanished under the dynamic electropulsing.

In comparison, the twins microstructure decreased gradually in the static EPT specimens [12], when the frequency increased from non-EPT to $209 \mathrm{~Hz}$ and 253 $\mathrm{Hz}$, and disappeared after the $294 \mathrm{~Hz}-\mathrm{EPT}$.

\subsection{Dislocation Dynamics}

The dynamic electropulsing induced dislocation evolution was detected by TEM examination. In the non-EPT specimen, there were plenty of dislocation arrays and nodes, which were formed during the previous rolling, as shown in Figure 3(b1). After $204 \mathrm{~Hz}-\mathrm{EPT}$ of dynamic electropulsing for about $10 \mathrm{~s}$, dislocation arrays and nodes decreased, as shown in Figure 3(b2). When the frequency of electropulsing increased to $309 \mathrm{~Hz}$, the amount of dislocation was further reduced, as shown in Figure 3(b3).

It is suggested that under electropulsing, electron wind formed and pushed the defects in the specimen, such as dislocations and atomic vacancies towards the grain boundaries, where accumulation and annihilation of dislocation occurred at the same time [5-8].

With increasing frequency of electropulsing, both the accumulation and the annihilation of dislocation increased and were in an adequate balance at the grain boundaries. Thus, the dislocation density decreased [10].

\subsection{Driving Force for Dynamic Electropulsing Induced Phase Transformation}

The driving force for phase transformation in the dynamic EPT alloy specimens consists mainly of three parts:

$$
\Delta G=\Delta G_{\text {chem }}+\Delta G_{\text {ep }}+\Delta G_{\text {stress }},
$$

where $\Delta G_{\text {chem }}$ is the chemical Gibbs free energy, $\Delta G_{\text {ep }}$ the electrpulsing induced Gibbs free energy and $\Delta G_{\text {stress }}$ the stress induced Gibbs free energy. In the present study, the electropulsing considerably accelerated the decom-



(a)

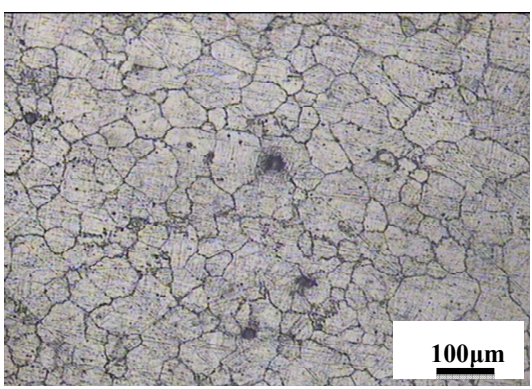

(b)

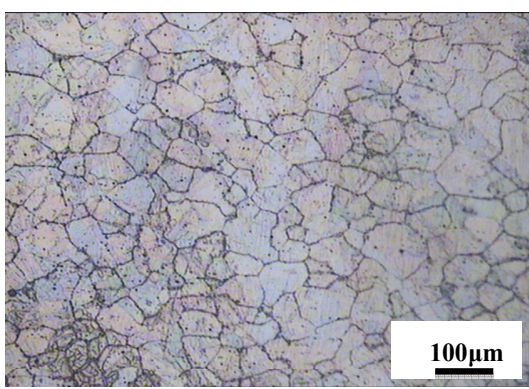

(c)

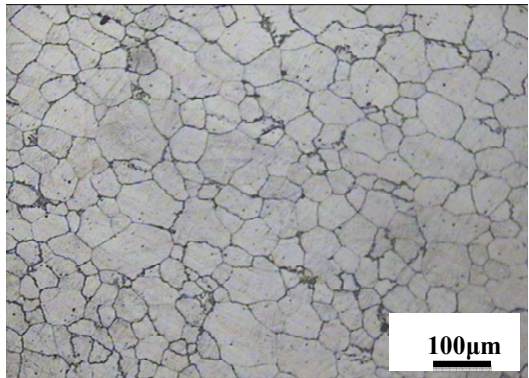

(d)



(e)

Figure 6. Optical micrographs of specimens: (a) Non-EPT and dynamic EPT with $126 \mathrm{~Hz}$ (b); $204 \mathrm{~Hz}$ (c); $265 \mathrm{~Hz}$ (d) and $309 \mathrm{~Hz}(\mathrm{e})$. 
position of $\beta$ phase in a way of up-quenching, i.e. the reverse phase transformations from final stable state back to a supersaturated state, $(\Delta G>0)$. For the reverse transformation, i.e. the decomposition of $\beta$ phase,

$\Delta G=\Delta G_{\text {ep }}+\Delta G_{\text {chem }}+\Delta G_{\text {stress }}$, where $\Delta G_{\text {chem }}$ appeared as an anti-driving force. The $\Delta G_{\text {ep }}$ became the driving force for the reverse transformation.

During dynamic electropulsing new structural distortions, such as dislocations and vacancies were created. When electron wind passed through the specimens, part of the electropulsing induced Gibbs free energy $\left(\Delta G_{\mathrm{ep}}\right)$ was exhausted through interaction between electrons and dislocation and vacancies which were created during mechanical deformation. Accordingly, the electropulsng induced $\Delta G_{\text {ep }}$ for the reverse phase transformation was reduced. In addition, during dynamic electropulsing, the rolling induced stress reduced instantaneously, and the residual stress induced $\Delta G_{\text {stress }}$ in the dynamic EPT specimen was less than that in the static rolled specimen.

Therefore, the total $\Delta G$ in the dynamic EPT specimen was smaller than that in the static EPT specimen, i.e. the dynamic electropulsing was less effective than the static electropulsing in accelerating decomposition of $\beta$ phase.

\subsection{Changes of Machining Properties Induced by EPT}

Shown in Figure 7 and Figure 8 are the SPDT cutting force and surface roughness of the non-EPT specimen and four dynamic EPT specimens, with various frequencies of electropulsing $(126 \mathrm{~Hz}, 204 \mathrm{~Hz}, 265 \mathrm{~Hz}$ and 309 $\mathrm{Hz}$ ), respectively. It can be seen that the mean cutting force decreased significantly when the frequency of electropulsing increased. For the non-EPT specimen, the mean cutting force was $67.4 \mathrm{mN}$, with the surface roughness of $16.2 \mathrm{~nm}$. When the frequency of electropulsing increased to $126 \mathrm{~Hz}$, the mean cutting force decreased to $46.2 \mathrm{mN}$, with the surface roughness of 15.8 $\mathrm{nm}$; upon the frequency being further increased to 204 $\mathrm{Hz}, 26 \mathrm{~Hz}$ and $309 \mathrm{~Hz}$, the mean cutting force decreased to $44.7 \mathrm{mN}, 49.8 \mathrm{mN}$, and $50.7 \mathrm{mn}$, with the surface roughness of $12.3 \mathrm{~nm}, 10.6 \mathrm{~nm}$ and $12.1 \mathrm{~nm}$, respectively. From Figure 5(b) and Figure 6, it can be seen that under dynamic electropulsing, dislocation density decreased, and large amounts of deformation twins diminished with a homogenous fine grain structure. As a result, the cutting force for SPDT processing decreased and surface roughness improved when the frequencies increased. The machinability of AZ91 alloy in SPDT was improved under dynamic electropulsing.

\section{Conclusions}

In summary, it is concluded as follows:

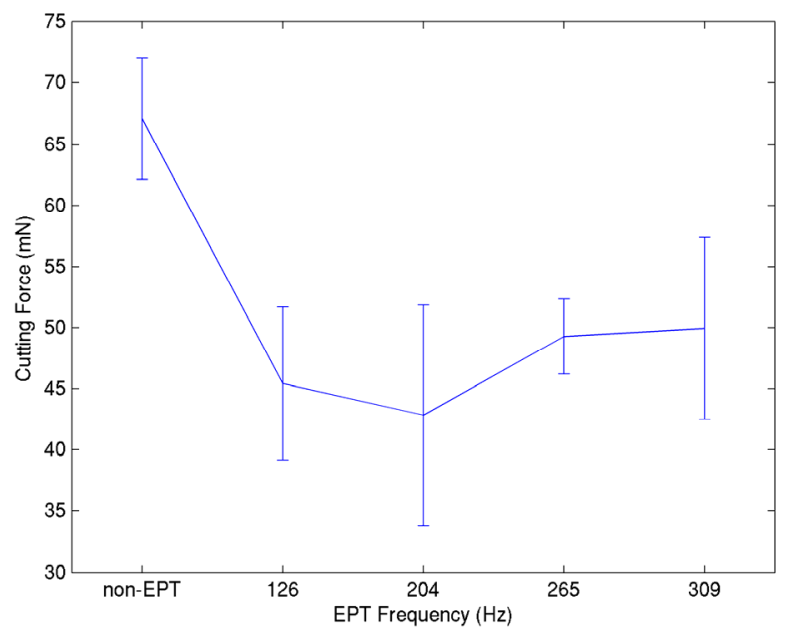

Figure 7. Mean cutting force under various frequencies of electropulsing with depth of cut of $20 \mu \mathrm{m}$.

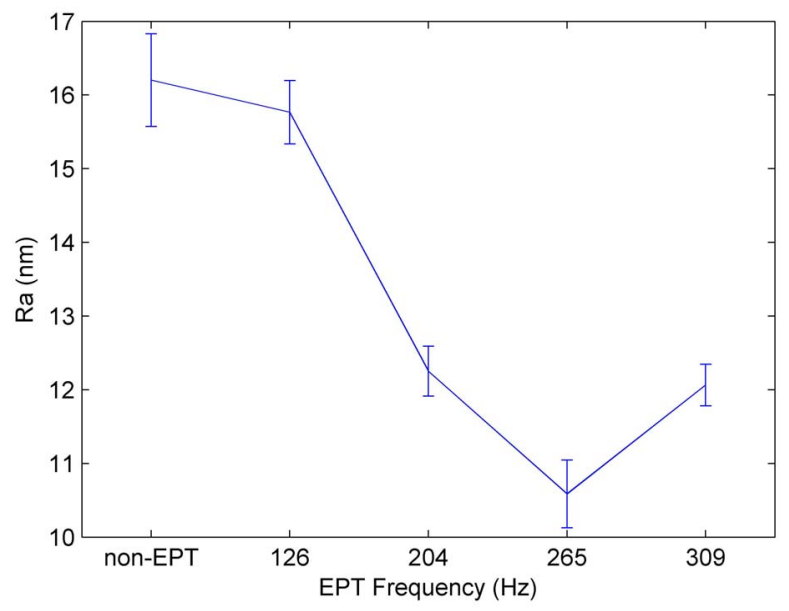

Figure 8. Surface roughness changes under different frequency of EPT, with depth of cut of $20 \mu \mathrm{m}$.

1) The $\beta$ phase decomposition was considerably accelerated by a way of up-quenching under dynamic electropulsing, compared with the conventional thermal processing. The dynamic electropulsing was less effective than the static electropulsing in accelerating decomposition of $\beta$ phase.

2) Under dynamic electropulsing, the twins microstructure disappeared in the rolled alloyAZ91, and a homogeneous fine grain structure was achieved. Dynamic electropulsing was more effective in reducing residual stress than the static electropulsing.

3) Under dynamic electropulsing, the SPDT cutting force decreased and surface roughness improved. The machinability of AZ91 alloy was improved.

\section{Acknowledgements}

The work described in this paper was partially supported 
by a grant from the Research Grants Council of the Hong Kong Special Administrative Region, China (Project No. PolyU 5316/09E).

\section{REFERENCES}

[1] Y. H. Zhu, "General Rule of Phase Decomposition in Zn-Al Based Alloys II-On Effect of External Stress on Phase Transformation," Materials Transactions, Vol. 45, No. 11, 2004, pp. 3083-3097. doi:10.2320/matertrans.45.3083

[2] O. A. Troitskii, "Electromechnical Effect in Metals," Journal of Experimental and Theoretical Physics, Vol. 10, 1969, pp. 18-22.

[3] H. Conrade and A. F. Sprecher, "Dislocation in Solid," Elsevier, Amsterdam, 1989, p. 497.

[4] H. Conrad, J. White, W. D. Cao, X. P. Lu and A. F. Sprecher, "Effect of Electric Current Pulses on Fatigue Characteristics of Polycrystalline Copper," Materials Science and Engineering: A, Vol. 145, No. 1, 1991, pp. 1-12. doi:10.1016/0921-5093(91)90290-4

[5] D. Yang and H. Conrade, "Exploratory Study into the Effects of an Electric Field and High Current Density Electropulsing on the Plastic Deformation of TiAl," Intermetallics, Vol. 9, 2001, pp. 943-947.

[6] J. R. Lloyd, "Electromigration in Integrated Circuit Conductors," Journal of Physics D: Applied Physics, Vol. 32, No. 17, 1999, pp. R109-R118. doi:10.1088/0022-3727/32/17/201
[7] S. H. Xiao, J. D. Guo and S. X. Li, "The Effect of Electropulsing on Dislocation Structures in [223] Coplanar Double-Slip-Oriented Fatigued Copper Single Crystals," Philisophycal Magazine Letters, Vol. 82, No. 11, 2002, pp. 617-622. doi:10.1080/0950083021000030397

[8] Y. Z. Zhou, W. Zhang, J. D. Guo and G. H. He, "Diffusive Phase Transformation in a Cu-Zn Alloy under Rapid Heating by Electropulsing," Philosophycal Magazine Letters, Vol. 84, No. 5, 2004, pp. 341-348. doi:10.1081/09500830410001664535

[9] V. V. Stolyarov, "Deformability and Nanostructuring of TiNi Shape-Memory Alloys during Electroplastic Rolling," Materials Science and Engineering: A, Vol. 503, No. 1-2, 2009, pp. 18-20. doi:10.1016/j.msea.2008.01.094

[10] Y. H. Zhu, S. To, W. B. Lee, X. M. Liu, Y. B. Jiang and G. Y. Tang, "Effect of Dynamic Electropulsing on Microstructure and Elongation of a Zn-Al Based Alloy," Materials Science and Engineering: A, Vol. 501, No. 1-2, 2009, pp. 125-132. doi:10.1016/j.msea.2008.09.080

[11] Y. B. Jiang, G. Y. Tang, C. H. Shek and Y. H. Zhu, "On the Thermodynamics and Kinetics of Electropulsing Induced Dissolution of $\beta-\mathrm{Mg}_{17} \mathrm{Al}_{12}$ Phase in an Aged Mg9Al-1Zn Alloy," Acta Materialia, Vol. 57, No. 16, 2009 , pp. 4797-4808.

[12] D. Zhang, S. To, Y. H. Zhu, H. Wang and G. Y. Tang, "Static Electropulsing Induced Microstructural Changes and Their Effect on the Ultra-Precision Machining of Cold-Rolled AZ91 Alloy," Metallurgical and Materials Transactions A, 2011, in press. 